\title{
Planar Helium Plasma J et Plasma "Bullets" Formation, 2D "Bullets" Concept and Imaging
}

\author{
Danil Dobrynin* \& Alexander Fridman \\ C\&J Nyheim Plasma Institute, Drexel University, Camden NJ 08103 \\ *Address all correspondence to: Danil Dobrynin, C\&J Nyheim Plasma Institute, Drexel University, Camden NJ 08103, \\ E-mail: danil@drexel.edu
}

\begin{abstract}
Applications of dielectric barrier discharge (DBD)-based atmospheric pressure plasma jets are often limited by the relatively small area of treatment due to their one-dimensional (1D) configuration. This letter describes the first results demonstrating generation of two-dimensional (2D) plasma "bullets" and 2D plasma jets that permit rapid treatment of large targets. Imaging of pulsed DBD in helium (He) and ionization wave propagation along the dielectric surface shows that DBD evolution starts with formation of transient anode glow; only then does the development of cathode-directed streamers continue. The anode glow can propagate as an ionization wave along the dielectric surface outside of the discharge gap. We show that plasma "bullet" propagation is not limited to $1 \mathrm{D}$ geometry (i.e., tubes) and that it can be organized in a planar plasma jet form or other 2D (or even 3D) shapes.
\end{abstract}

KEY WORDS: plasma jet, plasma bullets, fast imaging, pulsed atmospheric pressure plasma

Nonthermal atmospheric plasma jets (APPJ) based on dielectric barrier discharge (DBD) have attracted considerable interest in recent decades. ${ }^{1-3}$ Multiple research groups have studied these plasma sources for various applications like surface modification, ${ }^{5,6}$ decontamination, ${ }^{6-9}$ cancer treatment, ${ }^{10-12}$ and others. Numerous plasma diagnostics have been conducted to determine chemical and physical characteristics. One of the important challenges and limitations of existing one-dimensional (1D) plasma jets is related to the relatively small area of treatment. ${ }^{13-19}$ Here, we address this problem and offer a proposal to generate 2D plasma jets that allow rapid treatment of large surface-area targets.

Many reports have shown that plasma jets do in fact propagate via a so-called "plasma bullet," a train of discrete surface ionization waves traveling along dielectric surface (sometimes to address the ionization wave reconnection to the "parent" DBD plasma. These are called "pulsed atmospheric-pressure plasma streams" (PAPS). Although the physics of $1 \mathrm{D}$ jets and plasma bullet propagation has been described, ${ }^{20-30}$ the connection between the processes in the DBD plasma and bullet initiation is not yet clearly understood. Here, we demonstrate that plasma bullets originate from the initial anode glow (i.e., surface discharge) in DBD that precedes volumetric streamer discharge and propagates along the surface of dielectric to form the jet upon exiting the discharge chamber into the atmosphere. Based on this observation, we offer a possibility for rearranging the classical $1 \mathrm{D}$ tube configuration of APPJ into a 2D plasma jet. 
In these studies, we used a microsecond-pulsed high-voltage power supply with an output of $20 \mathrm{-kV}$, peak-to-peak, pulse duration of $\sim 8 \mu \mathrm{s}$ (Fig. 1a) and a frequency of $500 \mathrm{~Hz} .{ }^{31}$ Plasma jets were generated in helium (He) flow (99\%, Airgas). To monitor
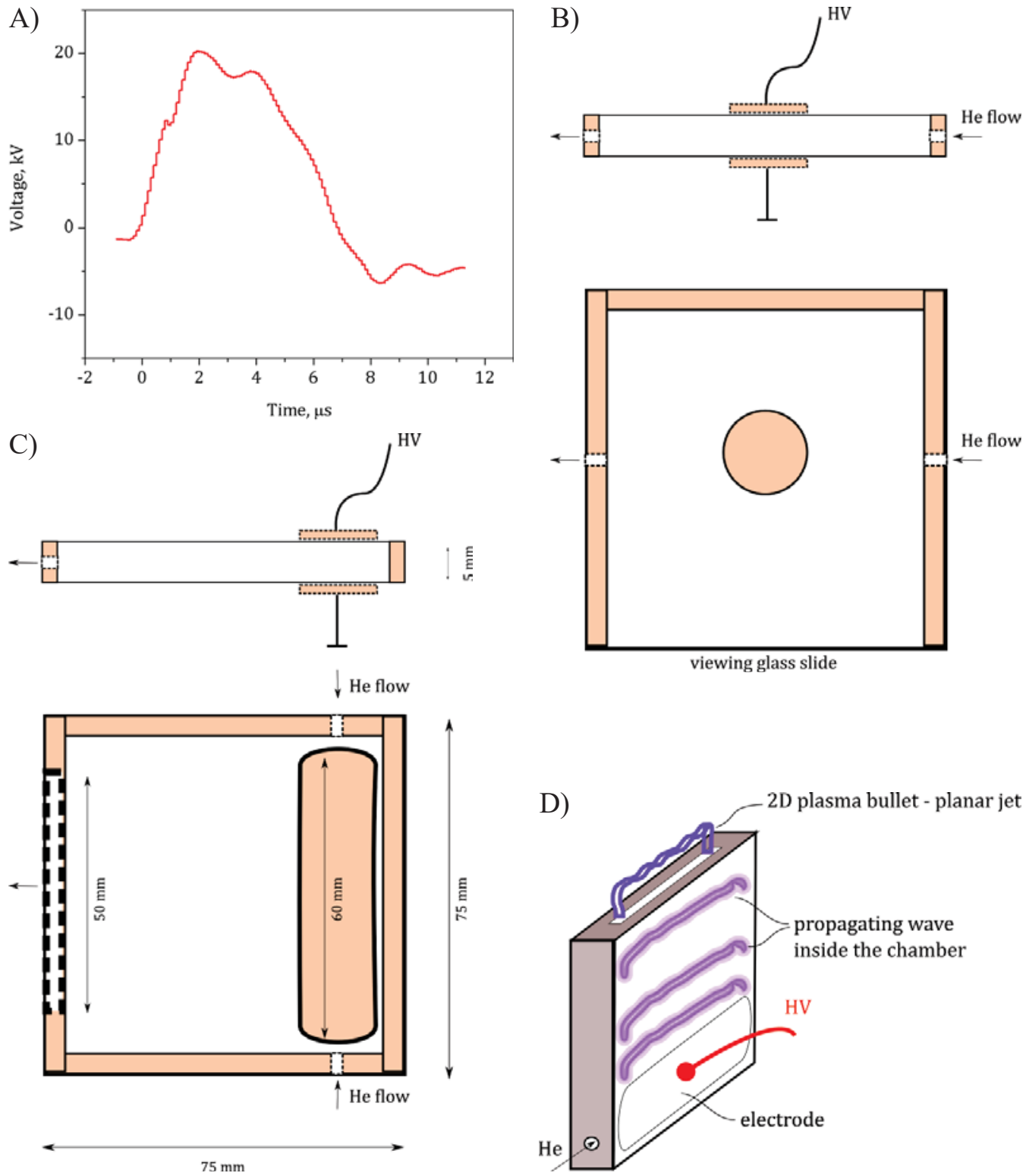

FIG. 1: Experimental setup: (a) Typical voltage waveform; (b) Discharge chamber schematic for visualization of surface ionization wave development and propagation; (c) Discharge chamber schematic for generation of planar plasma jet; and (d) Drawing of the planar plasma jet setup 
the discharge development and ionization wave propagation, we used a 4Picos ICCD camera from Stanford Computer Optics triggered using P6015A high-voltage probe (75 MHz bandwidth, Tektronix) also connected to a $1 \mathrm{GHz}$ DPO-4104B oscilloscope (Tektronix).

Two similar discharge chambers were used for generation and imaging of DBD, surface ionization waves, plasma "bullets," and the planar plasma jet. Both chambers were made of two 1 -mm-thick $75 \times 75$-mm glass slides separated by a 5 -mm-thick dielectric to form a rectangular cuboid. The first setup used to study DBD development and propagation of surface ionization waves employed a 1-mm-thick glass slide glued to the side of the chamber to visualize the discharge from the side (Fig. 1b). Here, we used two 20-mm-diameter electrodes fixed in the center of glass sides of the chamber, as well as two $2 \mathrm{~mm}$ holes for He flow though the setup at $\sim 0.5 \mathrm{~L} / \mathrm{m}$. To demonstrate the possibility of reconfiguring the 1D APPJ into a planar plasma jet, in the second chamber, instead of a circular gas outlet, we used a $1.5-\mathrm{mm}$-wide by $50-\mathrm{mm}$-long slit, as well as a $60-\mathrm{mm}$-long by $20-\mathrm{mm}$-wide copper electrodes fixed on the outer surfaces of the glass slides (Fig. 1c - d). Gas was supplied through two 2-mm holes at rate of $\sim 5 \mathrm{~L} / \mathrm{m}$.

Our first experiments aimed to increase our understanding of the development of plasma bullets. We employed a fast imaging technique using an ICCD camera triggered by a high-voltage pulse. During the high-voltage pulse, the discharge reignited several times (i.e., in a series of breakdowns), and we focused only on the first illumination. The DBD begins with the development of a series of avalanches traveling from the grounded electrode toward the powered anode. As the avalanche reaches the anode, the presence of the dielectric causes an accumulation of the charge, leading to the generation of electron densities (and local electric fields) sufficient to form a surface discharge that appears as a bright anode glow region (Fig. 2). This anode glow, or "pancake," is generated prior to the volumetric discharge that develops via traditional cathode-directed streamers. This series of events is not limited to the He atmosphere; in fact, similar discharge behaviors can also be seen for atmospheric air nanosecondpulsed DBD (e.g., Liu et al. ${ }^{32}$ ).
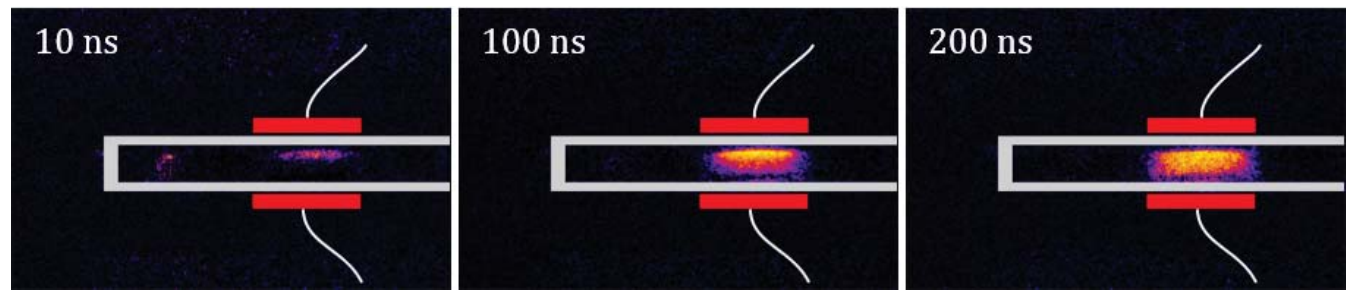

FIG. 2: Development of DBD in He: single-exposure ICCD images (false color) taken with 10 ns exposure time. Delay time was indicated after the trigger (when high-voltage pulse reached $\sim 5 \mathrm{kV}$ ). The faint glow on the first image in the left region of the chamber is an imaging artefact.

Volume 8, Issue 2, 2018 
Because the anode glow region can be viewed as a second capacitor (i.e., a third "electrode" near the anode), high electric fields at its edges facilitate the development of a surface wave that propagates along the surface of the dielectric. This phenom-enon is clearly depicted in Fig. 3 as a radially propagating ring with the velocity of $\sim 30 \mathrm{~km} / \mathrm{s}$; these images were taken from both side and top of the discharge chamber. As the wave reaches the output gas hole in the dielectric wall, it exits the discharge chamber in the form of a plasma "bullet." In this experiment, because of the discharge chamber configuration, the "bullet" most probably does not have a traditional "donut" shape, unlike tubular setups, in which the ionization wave propagates in the tube along its inner surface. ${ }^{3,33}$

Here, we have demonstrated the relation between the formation of the surface ionization wave in DBD and plasma "bullets." In this configuration, we were able to generate a 2D ionization surface wave; therefore, we propose a new configuration of APPJ to generate planar plasma jets. For this demonstration, we used the second discharge chamber with

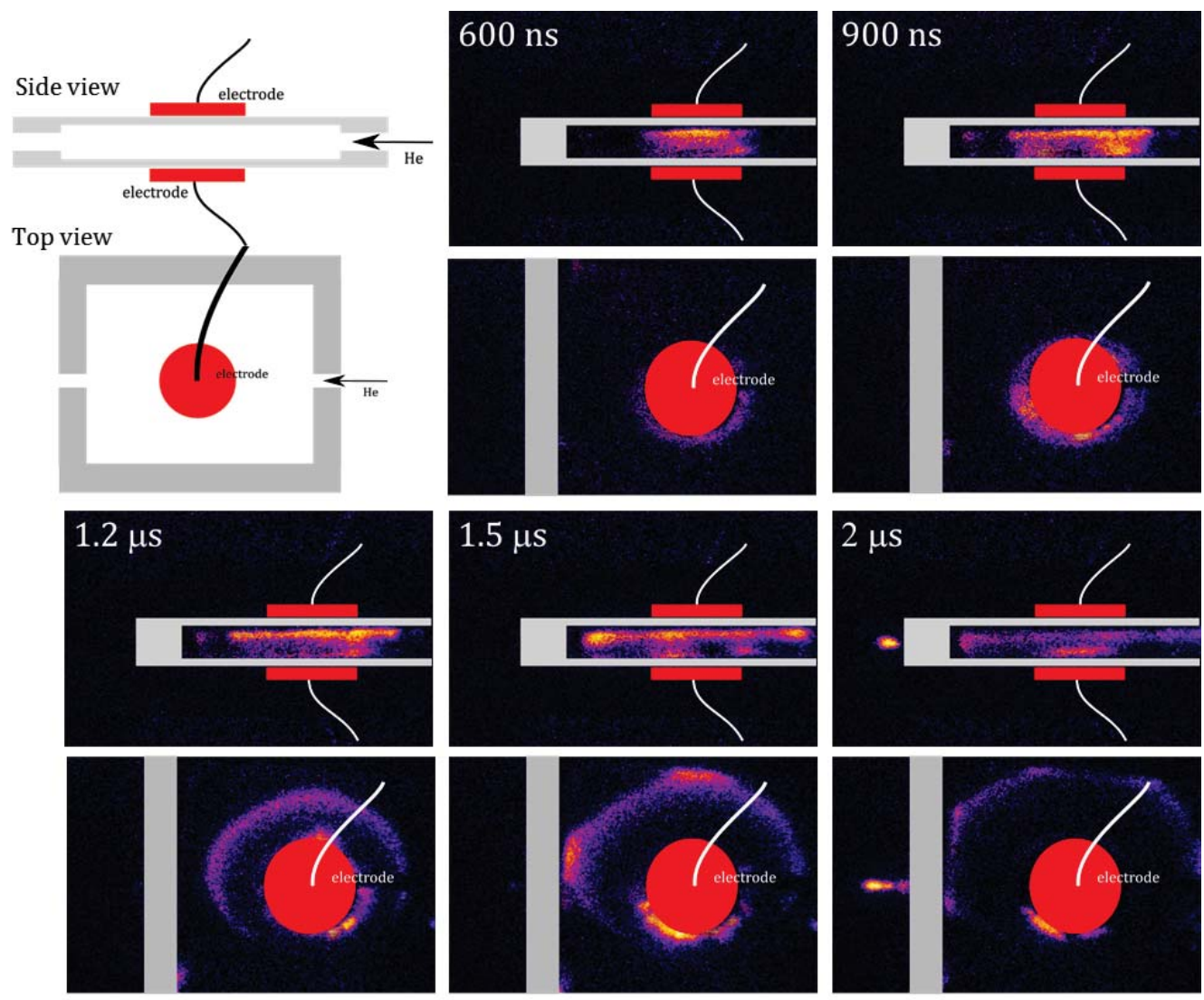

FIG. 3: Propagation of surface ionization wave: single-exposure ICCD images (false color) taken with 300 ns exposure time. Delay time was indicated after the trigger (when high-voltage pulse reached $\sim 5 \mathrm{kV}$ ). Separate images were taken for the top and side views. 
exit gas opening in the form of a long slit. In these experiments, similar to the first case, the propagation of ionization wave inside the chamber along the dielectric surface is clearly evident (Fig. 4). As the wave reaches the exit opening slit, it forms a planar plasma jet that propagates into the outer atmosphere. However, due to flow conditions that cause effective mixing with air, and possibly low specific energy input, the propagation length of the jet is only $\sim 1 \mathrm{~cm}$ or less. In principle, the output gas opening here is not limited to a straight planar slit and can be organized in a more complex shape (e.g., a wave-shaped slit); thus, it is possible to generate plasma jets with various complex 3D profiles. Similarly, the width of the jet can be controlled by the distance from the electrodes and, in general, is limited only by the pulse energy provided by the power supply. It is possible, therefore, to generate jets with any width to accommodate treatment of large targets.

The photographs in Fig. 5 demonstrate possible applications for treatment: attachment of the jet to a floating insulated wire, and a finger. Although the distance from the discharge chamber output opening to the target is not constant and varies from $\sim 0.5$ to 1 $\mathrm{cm}$, the plasma jet allows relatively uniform treatment. Also, because of the conductance of the tissue (floating potential), the plasma jet can propagate to a longer length. More detailed studies are needed to explore this phenomena and 3D plasma jet generation method in detail.

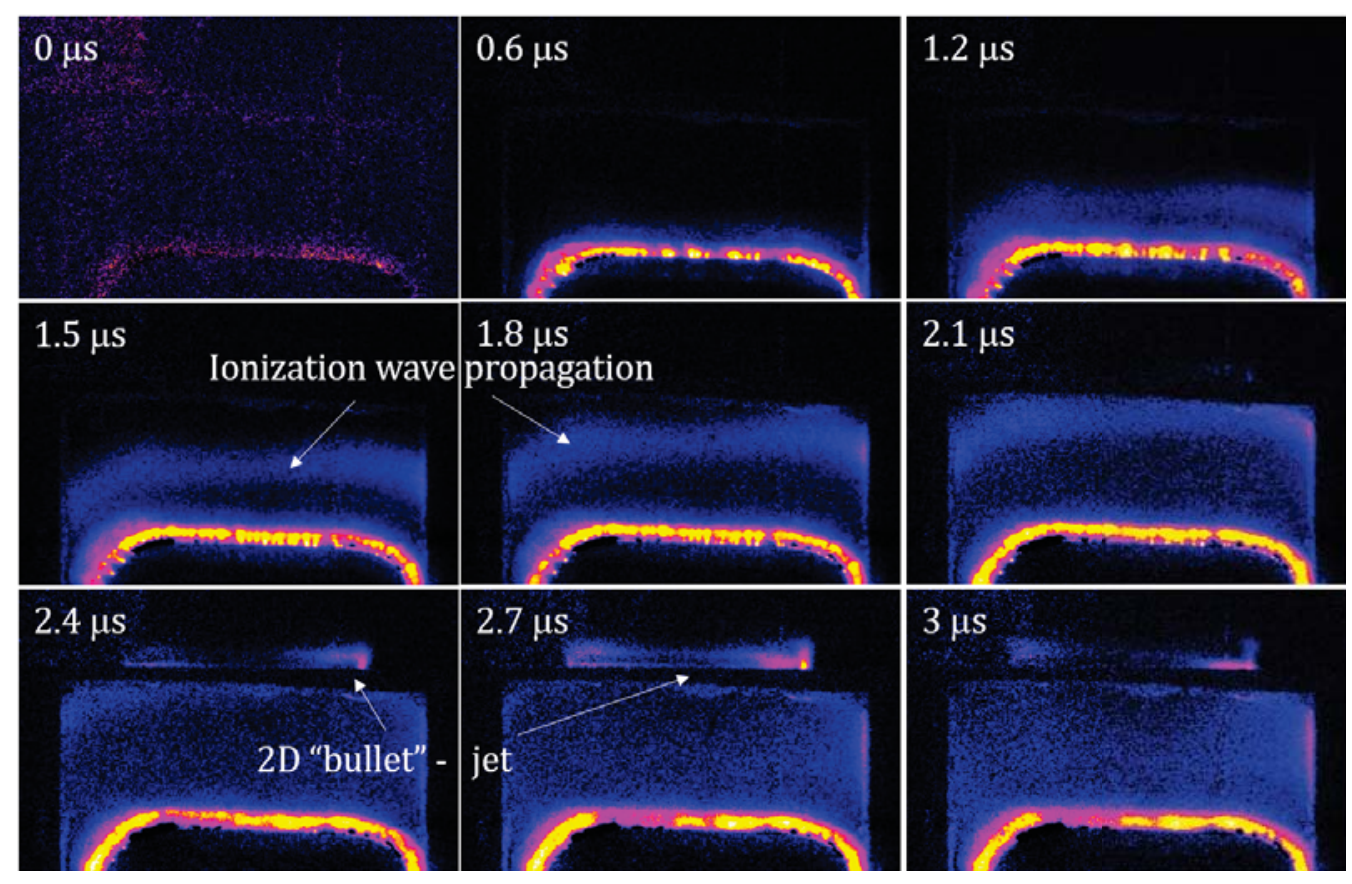

FIG. 4: Propagation of surface ionization wave and formation of planar plasma jet: single-exposure ICCD images (false color) taken with $300 \mathrm{~ns}$ exposure time. Delay time was indicated after the trigger (when high voltage pulse reached $\sim 5 \mathrm{kV}$ ).

Volume 8, Issue 2, 2018 

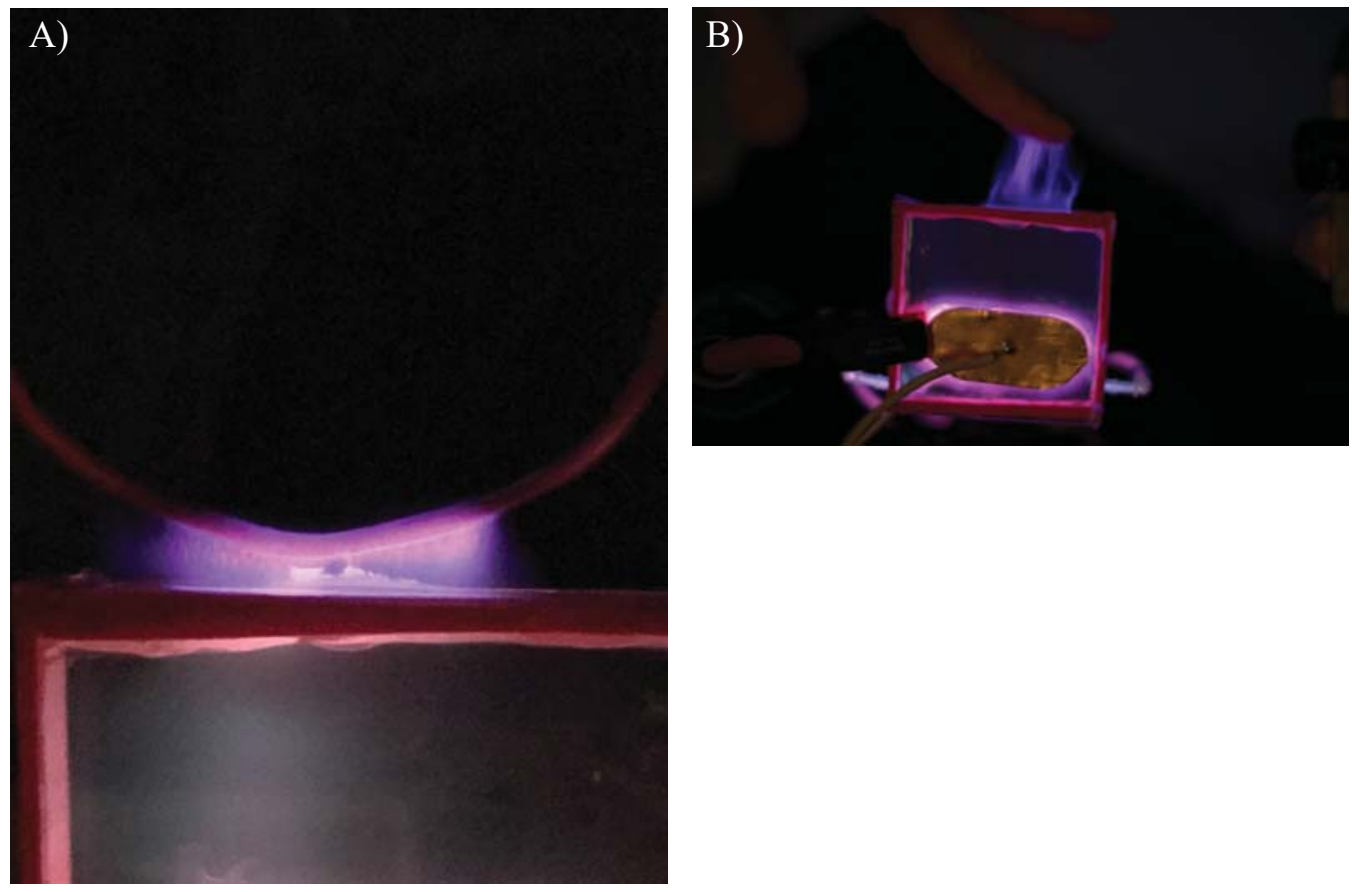

FIG. 5: Photographs of planar plasma jet applications to a floating potential insulated wire and a finger

Moreover, imaging of pulsed DBD in He and ionization wave propagation along the dielectric surface leads us to the following conclusions:

1. The evolution of pulsed DBD plasma starts with the formation of a transient anode glow, which only then continues with development of cathode-directed streamers.

2. In the case of noble gases, the anode glow can propagate as an ionization wave along the dielectric surface outside the discharge gap. For traditional dielectric tubes, the ionization wave appears as a "plasma bullet," often with a characteristic "donut" shape.

3. Plasma bullet propagation is not limited to $1 \mathrm{D}$ geometry (tubes) and can be organized in a planar jet form or other 2D (or even 3D) shapes.

4. Propagation of the $2 \mathrm{D}$ plasma bullet results in generation of $2 \mathrm{D}$ (or even 3D) plasma jets. The size of these jets is limited by the pulse energy and gas flow characteristics.

Plasma jets with complex 2D (or 3D) geometries open new possibilities for large-surface material processing and plasma medicine. 


\section{ACKNOWHEGMENT}

This work was partially supported by the NSF/DOE Partnership in Basic Plasma Science and Engineering grant (DOE Grant No. DE-SC0016492, PI: D. Dobrynin).

\section{REFERENCES}

1. Laroussi M, Lu X, Keidar M. Perspective: the physics, diagnostics, and applications of atmospheric pressure low temperature plasma sources used in plasma medicine. J Appl Phys. 2017;122:020901.

2. Winter J, Brandenburg R, Weltmann K-D. Atmospheric pressure plasma jets: an overview of devices and new directions. Plasma Source Sci Technol. 2015;24:64001.

3. Lu X, Laroussi M, Puech V. On atmospheric-pressure non-equilibrium plasma jets and plasma bullets. Plasma Source Sci Technol. 2012;21:034005.

4. Babayan S E, Jeong JY, Tu VJ, Park J, Selwyn GS, Hicks RF. Deposition of silicon dioxide films with an atmospheric-pressure plasma jet. Plasma Source Sci Technol. 1998;7:286.

5. Fanelli F, Fracassia F. Atmospheric pressure non-equilibrium plasma jet technology: general features, specificities and applications in surface processing of materials. Surf Coat Technol. 2017;322:174201.

6. Ehlbeck J, Schnabel U, Polak M, Winter J, Woedtke Th von, Brandenburg R, Hagen T von dem, Weltmann K. Low temperature atmospheric pressure plasma sources for microbial decontamination. J Phys D Appl Phys. 2011;44:013002.

7. Laroussi M, Tendero C, Lu X, Alla S, Hynes WL. Inactivation of bacteria by the plasma pencil. Plasma Processes Polym. 2006;3:470-3.

8. Laroussi M. Low-temperature plasma jet for biomedical applications: a review. IEEE Trans Plasma Sci. 2015;43(3):703.

9. Laroussi M, Karakas E, Hynes W. Influence of cell type, initial concentration, and medium on the inactivation efficiency of low-temperature plasma. IEEE Trans Plasma Sci. 2011;39(11):2960-1.

10. Barekzi N, Laroussi M. Effects of low temperature plasmas on cancer cells. Plasma Proc Polym. 2013;10(12):1039-50.

11. Keidar M. Cold atmospheric plasma in cancer therapy. Phys Plasma. 2013;20(5):057101.

12. Vandamme M, Robert E, Sobilo J, Sarron V, Ries D, Dozias S, Legrain B, Lerondel S, Pape AL, Pouvesle JM. In situ application of nonthermal plasma: preliminary investigations for colorectal and lung tolerance. Proc 20th Int Symp Plasma Chem. 2011:1-4.

13. Ghasemi M, Olszewski P, Bradley JW, Walsh JL. Interaction of multiple plasma plumes in an atmospheric pressure plasma jet array. J Phys D Appl Phys. 2013;46:52001.

14. Nie QY, Cao Z, Ren CS, Wang DZ, Kong MG. A two-dimensional cold atmospheric plasma jet array for uniform treatment of large-area surfaces for plasma medicine. New J Phys. 2009; $11: 115015$.

15. Babaeva NY, Kushner MJ. Interaction of multiple atmospheric-pressure micro-plasma jets in small arrays: He/O2 into humid air. Plasma Source Sci Technol. 2014;23:015007.

16. Cao Z, Walsh JL, Kong MG. Atmospheric plasma jet array in parallel electric and gas flow fields for three-dimensional surface treatment. Appl Phys Lett. 2009;94:021501.

17. Foest R, Kindel E, Ohl A, Stieber M, Weltmann KD. Non-thermal atmospheric pressure discharges for surface modification. Plasma Phys Control Fusion. 2005;47:B525.

18. Ma JH, Shih DC, Park S-J, Eden JG. Microplasma jets generated by arrays of microchannels fabricated in flexible molded plastic. IEEE Trans Plasma Sci. 2011;39:2700.

19. Robert E, Darny T, Dozias S, Iseni S, Pouvesle JM. New insights on the propagation of pulsed atmospheric plasma streams: from single jet to multi jet arrays. Phys Plasma. 2015;22:122007.

Volume 8, Issue 2, 2018 
20. Begum A, Laroussi M, Pervez MR. Atmospheric pressure He-air plasma jet: breakdown process and propagation phenomenon. AIP Adv. 2013;3:062117.

21. Lu X, Naidis GV, Laroussi M, Ostrikov K. Guided ionization waves: theory and experiments. Phys Rep. 2014;540:123-166.

22. Teschke M, Kedzierski J, Finantu-Dinu EG, Korzec D, Engemann J. High-speed photographs of a dielectric barrier atmospheric pressure plasma jet. IEEE Trans Plasma Sci. 2005;33(2):310-1.

23. Murakami T, Niemi K, Gans T, O'Connell D, Graham WG. Chemical kinetics and reactive species in atmospheric pressure helium-oxygen plasmas with humid-air impurities. Plasma Sources Sci Technol. 2013;22:15003.

24. Darny T, Pouvesle JM, Puech V, Douat C, Dozias S, Robert E. Analysis of conductive target influence in plasma jet experiments through helium metastable and electric field measurements. Plasma Sources Sci Technol. 2017;26:45008.

25. Boselli M, Colombo V, Ghedini E, Gherardi M, Laurita R, Liguori A, Sanibondi P, Stancampiano A. Schlieren high-speed imaging of a nanosecond pulsed atmospheric pressure non-equilibrium plasma jet. Plasma Chem Plasma Process. 2014;34:853-69.

26. Robert E, Sarron V, Riès D, Dozias S, Vandamme M, Pouvesle JM. Characterization of pulsed atmospheric pressure plasma streams (PAPS) generated by a plasma gun. Plasma Sources Sci Technol. 2012;21:34017.

27. Bourdon A, Darny T, Pechereau F, Pouvesle J-M, Viegas P, Iséni S, Robert E. Numerical and experimental study of the dynamics of a $\mu$ s helium plasma gun discharge with various amounts of N2 admixture. Plasma Sources Sci Technol. 2016;25:35002.

28. Darny T, Pouvesle JM, Puech V, Douat C, Dozias S, Robert E. Analysis of conductive target influence in plasma jet experiments through helium metastable and electric field measurements. Plasma Sources Sci Technol. 2017;26:105001.

29. Boeuf JP, Yang LL, Pitchford LC. Dynamics of a guided streamer ('plasma bullet') in a helium jet in air at atmospheric pressure. J Phys D Appl Phys. 2013;46:015201.

30. Jarrige J, Laroussi M, Karakas E. Formation and dynamics of plasma bullets in a non-thermal plasma jet: influence of the high-voltage parameters on the plume characteristics. Plasma Sources Sci Technol. 2010;19:065005.

31. Park D, Fridman G, Fridman A, Dobrynin D. Plasma bullets propagation inside of agarose tissue model. IEEE Trans Plasma Sci. 2013;41(7):1725.

32. Liu C, Dobrynin D, Fridman A. Uniform and non-uniform modes of nanosecond-pulsed dielectric barrier discharge in atmospheric air: fast imaging and spectroscopic measurements of electric fields. J Phys D Appl Phys. 2014;47:252003.

33. Mericam-Bourdet N, Laroussi M, Begum A, Karakas E. Experimental investigations of plasma bullets. J Phys D Appl Phys. 2009;42:055207. 